\title{
Substituição de fatores produtivos na produção de soja no Brasil ${ }^{1}$
}

\author{
Luciane Conte* \\ Joaquim Bento de Souza Ferreira Filho**
}

Resumo: O objetivo desse trabalho é analisar as inter-relações entre os principais insumos utilizados no processo produtivo da soja e as suas possibilidades de substituição, através dos conceitos das elasticidades-preço da demanda, de Allen e de Morishima. O referencial teórico do estudo é o da teoria da dualidade da função custo e da função de produção. Os dados são provenientes de uma pesquisa de campo realizada nos cinco principais estados produtores do País. Químicos, capital, terra, mão-deobra e outros custos foram as variáveis analisadas. As elasticidades-preço cruzadas mostraram que há complementaridade entre os fatores mão-deobra e capital. As elasticidades de substituição parcial de Allen indicaram substituição entre a maior parte dos fatores de produção. Houve uma forte relação de complementaridade entre os fatores capital e mão-de-obra e de substituição entre os fatores químicos e mão-de-obra. Na classificação de Morishima, capital e mão-de-obra são complementares quando o preço de capital varia e substitutos quando varia o preço do fator mão-de-obra.

Palavras-chave: função custo, elasticidade de substituição, soja, mercado de fatores; teoria da dualidade.

\footnotetext{
${ }^{1}$ Os autores agradecem à Fundação de Amparo à Pesquisa do Estado de São Paulo (FAPESP) pelo financiamento do projeto de pesquisa.

* Doutor, pesquisadora da Lentikia Capital (Hedge Fund). 33 Cavendish Square London W1G 0PW. Inglaterra. ktzconte@yahoo.com.br

** Professor Associado, Escola Superior de Agricultura “Luiz de Queiroz” - ESALQ, - USP. Depto. de Economia, Administração e Sociologia. Piracicaba, SP. jbsferre@esalq.usp.br
} 


\section{Classificação JEL: Q12; C31; D24}

Abstract: This paper analyzes the substitution relations between the main inputs used in soybean production in Brazil, through the estimates of Allen and Morishima substitution elasticities. The theoretical approach used is the production/cost duality. The data was obtained from field research in the five main production states in the country. Chemicals, capital, land, labor and other costs were the variables under study. The estimated cross-price elasticities pointed to complementary relations between labor and capital. The Allen partial substitution elasticities showed substitution between most of the production factors, but a strong complementarity relation was found between capital and labor. In the Morishima elasticity of substitution concept capital and labor were found to be complements when the price of capital varies and substitutes when the price of labor varies.

Key words: cost function, elasticity of substitution, soybean, factor markets, duality.

JEL Classification: Q12; C31; D24

\section{Introdução}

A importância da agricultura para o crescimento econômico de um país sempre foi um tópico de grande relevância dentro da teoria econômica. Em um país em desenvolvimento como o Brasil, a importância do agronegócio é bastante significativa. Em 2004, o PIB do agronegócio brasileiro, que inclui desde a produção primária até a indústria de processamento, insumos e serviços, foi de R \$ 533,98 bilhões, representando 30,07\% do PIB nacional, segundo dados da Confederação Nacional da Agricultura e Pecuária e do Centro de Estudos Avançados em Economia Aplicada da Universidade de São Paulo (CNA/CEPEA-USP, 2005). O PIB do setor agrícola brasileiro foi de R $\$ 379,89$ bilhões, contribuindo com 21,39\% na formação do PIB nacional.

A cadeia produtiva da soja é responsável por uma parcela significa- 
tiva do PIB do agronegócio brasileiro. Ela é composta por empresas produtoras de máquinas, equipamentos e insumos agrícolas, por propriedades agrícolas produtoras de soja em grão, por indústrias de processamento como esmagadoras e refinarias que produzem óleo e farelo de soja, e todos os demais agentes - exportadores, atacadistas, varejistas, entre outros - que operam na produção e distribuição de soja em grão e derivados (FIGUEIREDO, 2003). A indústria da soja é importante atualmente tanto para a captação de moedas estrangeiras pelas exportações brasileiras de farelo, óleo e grãos, como para o suprimento do mercado interno de óleos comestíveis e concentrados protéicos. O Brasil ocupa a posição de segundo maior produtor mundial de soja, sendo responsável por aproximadamente $24 \%$ da oferta global do produto (FAO, 2005). Atualmente, os líderes mundiais na produção de soja são os Estados Unidos, Brasil, Argentina, China, Índia e Paraguai.

A soja é um dos principais produtos da pauta de exportações brasileiras (BRASIL, 2005). Em 2004, o País exportou aproximadamente 19,25 milhões de toneladas de soja em grão, 14,48 milhões de farelo de soja e 2,50 milhões de óleo bruto e refinado. As exportações de soja representam aproximadamente $25,75 \%$ das exportações do agronegócio brasileiro, e em torno de 10,41\% de nossas exportações totais, segundo estatísticas da Companhia Nacional do Abastecimento (CONAB, 2005).

O objetivo deste trabalho foi identificar a estrutura de demanda de fatores de produção da sojicultura brasileira. Buscou-se caracterizar a interdependência no mercado de fatores e as possibilidades de substituição dos recursos no processo produtivo da atividade. A análise da tecnologia foi feita através da estimação das elasticidades cruzadas, de Allen e de Morishima.

\section{Metodologia}

O referencial teórico usado nesse estudo foi o da dualidade existente entre as funções de produção e custo, que estabelece que as informações economicamente relevantes referentes ao processo de produção podem ser obtidas a partir da função custo (CHRISTENSEN; GREENE, 1976; CHAMBERS, 1998; ALVES, 1995; BAIRAM, 1998; BAIRAM; KALYA, 1998). Para analisar as características dos insumos empregados na produção de soja no Brasil utilizou-se a forma funcional flexível 
translog para a função custo, assumindo que esta expressa o custo de produção como uma função dos preços dos insumos e da quantidade produzida (LERDA, 1979; GOMES; ROSADO, 2005):

$$
C^{*}=c\left(Y, P_{1}, P_{2}, \ldots, P_{n}\right)
$$

onde $\mathrm{C}^{*}$ é o custo mínimo de produção, Y é o nível de produção e $\mathrm{P}_{\mathrm{n}}$ é o preço dos insumos necessários ao nível de produção $\mathrm{Y}$.

Logaritmizando-se a expressão (1) e expandindo-a através da série de Taylor de segunda ordem em torno de um vetor unitário, obtém-se a função custo transcendental logarítmica (translog), indicada na expressão seguinte:

$$
\ln C^{*}=\alpha_{0}+\alpha_{Y} \ln Y+\sum_{i}^{n} \alpha_{i} \ln P_{i}+\frac{1}{2} \sum_{i}^{n} \sum_{j}^{n} \beta_{i j} \ln P_{i} \ln P_{j}+\sum_{i}^{n} \beta_{i Y} \ln P_{i} \ln Y+\frac{1}{2} \alpha_{Y Y}(\ln Y)^{2}
$$

onde os $\alpha$ são os parâmetros estruturais da função de custo e os índices $i$ e $j$ se referem aos fatores de produção utilizados na estimação da função custo.

A igualdade das derivadas parciais cruzadas, segundo o Teorema de Young, implica em uma restrição de simetria aos parâmetros estruturais da função custo translog, resultando em $\beta_{i j}=\beta_{i j}$ para todo $i, j$ ( $i$ \# $j$ ), enquanto a condição de homogeneidade linear da função no vetor de preços requer que (LERNER, 1934; LIMA, 2000):

$$
\sum_{i}^{n} \alpha_{i}=1 \text { e } \sum_{i}^{n} \beta_{i Y}=\sum_{i}^{n}{ }_{i j}=\sum_{i}^{n} \alpha_{i Y}=0
$$

A função custo translog deve atender localmente a duas propriedades importantes da função custo: monotonicidade e concavidade. A monotonicidade da função será satisfeita se as parcelas de custo forem não-negativas, enquanto a concavidade será atendida se o hessiano da matriz resultante for semidefinido negativo. Satisfeitas estas condições, as funções demanda podem ser estimadas através da aplicação do lema de Shephard (BEATTIE; TAYLOR, 1985), segundo o qual a derivada parcial da função custo translog em relação ao preço do insumo é igual à quantidade demandada do fator considerado, ou seja: 
$\frac{\partial \mathrm{h} C^{*}}{\partial \mathrm{h} p_{i}}=\frac{\partial C^{*}}{\partial P_{i}} \frac{P_{i}}{C^{*}}$

segundo o lema de Shephard $\frac{\partial C^{*}}{\partial P_{i}}=X_{i}$ vem que:

$$
\frac{\partial \mathrm{h} C^{*}}{\partial \mathrm{h} p_{i}}=\frac{P_{i} X_{i}}{C^{*}}=S_{i}
$$

onde $S_{i}$ corresponde à parcela dos custos relacionada ao i-ésimo insumo.

Tomando as derivadas parciais de (2) em relação a cada fator, resulta em:

$$
\frac{\partial \ln C^{*}}{\partial \ln p_{i}}=\alpha_{i}+\sum_{i}^{n} \beta_{i Y} \ln Y+\ln P_{j}=S_{i}
$$

que constitui um sistema de n-equações de parcelas de custo. A solução desse sistema de equações fornecerá os parâmetros estruturais da função custo necessários ao cálculo das elasticidades, como proposto por Binswager (1974).

As elasticidades-preço diretas $\left(\eta_{i i}\right)$ e cruzadas $\left(\eta_{i j}\right)$ são definidas como (BLACKORBY; RUSSEL, 1981; DIAS, 1982):

$$
\begin{aligned}
& \eta_{i i}=\frac{\beta_{i i}}{S_{i}}+S_{i}-1 \\
& \eta_{i j}=\frac{\beta_{i j}}{S_{i}}+S_{j}
\end{aligned}
$$

As elasticidades de substituição parcial de Allen $\left(\sigma_{i j}\right)$ podem ser definidas como:

$$
\begin{aligned}
& \sigma_{i i}=\frac{1}{S^{2}{ }_{i}}\left(\beta_{i i}+S^{2}{ }_{i}-S_{1}\right) \\
& \sigma_{i j}=\frac{1}{S_{i} S_{j}} \beta_{i j}+1
\end{aligned}
$$

Podemos ainda definir uma outra medida de substituibilidade, denominada de elasticidade de substituição de Morishima $\left(\sigma^{M}{ }_{i j}\right)$, conforme apresentada em Chambers (1998):

$$
\sigma^{M}{ }_{i j}=S_{j}\left(\sigma_{i j}-\sigma_{j j}\right)=\eta_{i j}-\eta_{j j}
$$


Merecem destaques alguns pontos importantes nas relações de substituibilidade. Primeiro, devemos destacar que pela homogeneidade da demanda de insumo, temos que $\sum_{i \# j} \sigma_{i j}=-\sigma_{j j}$ e pela concavidade da função custo devemos ter $\sigma_{j j} \leq 0$, logo $\sum_{i \# j} \sigma_{i j} \geq 0$ evidenciando que determinado insumo não pode ser Allen complementar a todos os demais insumos. Segundo, a elasticidade de Allen apresenta simetria, ou seja, $\sigma_{i j}=\sigma_{j i}$, o mesmo não ocorrendo com a elasticidade de Morishima. Por fim, quando dois insumos são Allen substitutos, $\left(\sigma_{i j}>0\right)$, eles também devem ser Morishima substitutos, $\left(\sigma^{M}{ }_{i j}>0\right)$. Entretanto, se eles forem complementares em Allen, $\left(\sigma_{i j}<0\right)$, não necessariamente serão Morishima complementares $\left(\sigma^{M}{ }_{i j}<0\right)$, pois pode ocorrer que $\left|\sigma_{i j}\right|<\left|\sigma_{j j}\right|$ e assim, eles serão substitutos pela definição de elasticidade de substituição de Morishima.

A significância dos valores obtidos para as elasticidades será avaliada pelos erros padrões ( $\mathrm{Se}$ ) para cada elasticidade, conforme definido por Binswager (1974), onde:

$$
\begin{aligned}
& S e_{\eta_{i j}}=\frac{S e_{\beta_{i j}}}{S} \\
& t=\frac{\eta_{i j}}{S e_{\eta_{i j}}}
\end{aligned}
$$

\subsection{Procedimentos de estimação}

O procedimento utilizado baseou-se na estimação de alguns dos parâmetros da função de custo total translog, a partir do sistema de “n” equações de parcelas de custo. Como os erros destas equações podem estar contemporaneamente correlacionados, o método utilizado na estimação deste sistema é o proposto por Zellner (1962), para equações aparentemente não-relacionadas (Seemingly Unrelated Regression - SUR). Além disto, uma vez que a soma das parcelas de custos é igual a um (1), torna-se necessário suprimir uma das equações do sistema a fim de evitar a singularidade da matriz de variâncias e covariâncias. 
Esta metodologia, utilizada em trabalhos como os de Berndt; Wood (1975), Zagatto (1991), Reis; Teixeira (1995), Rochelle; Ferreira Filho (1999); Ray (1982), é particularmente útil quando o objetivo da análise é o de se verificar as relações no mercado de fatores, mediante estimativa das elasticidades de substituição e elasticidades-preço direta e cruzada da demanda por fatores. Para tornar-se o modelo operacional, impõem-se as restrições teóricas de simetria e de homogeneidade, através da normalização da função de custo total translog. O problema da singularidade da matriz de variância e covariância dos erros das equações de parcela de custos é equacionado, deixando-se de fora a equação de parcela de custos de um dos fatores de produção. Estima-se, assim, o restante do sistema e obtêm-se os parâmetros excluídos por diferença.

\subsection{Caracterização sócio-econômica das diferentes regióes produtoras de soja}

Os dados utilizados nesse estudo foram obtidos através de uma pesquisa de campo, realizada no período de agosto a dezembro de 2005, para o levantamento de informações dos sistemas de produção de soja em grãos no Brasil, referentes à safra 2004/2005.

Aplicaram-se questionários em uma amostra de 218 sojicultores dos principais estados produtores do país. O objetivo foi a obtenção das variáveis econômicas e sociais que caracterizassem os produtores de cada região. Realizou-se a pesquisa em cooperativas, sindicatos rurais e revendas de produtos agrícolas. À medida que os produtores entravam nesses estabelecimentos, eles eram abordados e convidados a responder o questionário.

Duas grandes regiões brasileiras foram amostradas: Sul e CentroOeste. Escolheram-se as regiões que são pólos representativos da produção de soja dentro de cada estado. Na região Sul, aplicaram-se 79 questionários no Rio Grande do Sul e 43 no Paraná; na região CentroOeste entrevistaram-se 44 produtores no Estado do Mato Grosso do Sul, 29 produtores no Mato Grosso e 23 produtores em Goiás. No Estado do Mato Grosso a pesquisa foi realizada nas regiões de Lucas do Rio Verde, Sorriso, Campo Novo do Parecis e Rondonópolis; no Mato Grosso Sul amostraram-se propriedades nas regiões de Dourados, Maracaju, Sidrolândia e São Gabriel do Oeste; em Goiás as regiões escolhidas fo- 
ram as de Rio Verde e Jataí. Na região Sul, no Estado do Paraná foram amostradas quatro regiões: Londrina, Maringá, Cascavel e Ponta Grossa; no Estado do Rio Grande do Sul, as regiões foram as de Passo Fundo, Carazinho, Não-Me-Toque, Ijuí e Santa Rosa.

\subsection{Descrição das variáveis}

Para a análise dos custos de produção considerou-se desde o preparo do solo para o plantio até a colheita da soja. Não foram incluídos os gastos com transporte após a colheita, de armazenagem e comercialização dos grãos de soja. As variáveis consideradas foram: químicos (Q), capital $(\mathrm{K})$, mão-de-obra (L), terra (T) e outros custos (O). Os preços dos fatores foram obtidos diretamente, através da pesquisa, ou pela razão entre a despesa com o fator e a quantidade utilizada do mesmo. Para o Estado do Rio Grande do Sul considerou-se a média de produtividade de cada propriedade produtora de soja dos últimos três anos. Esse procedimento foi adotado em virtude da queda de produção, na safra 2004/2005, em decorrência da estiagem. Durante as entrevistas teve-se a cautela de perguntar se os demais procedimentos de manejo foram alterados. No entanto quando ocorreu a estiagem, a maior parte das pulverizações com herbicidas e defensivos agrícolas já havia sido realizada.

\subsubsection{Capital (K)}

O preço do capital foi determinado através da relação entre os fluxos de serviço do capital e o estoque de capital fixo (DIAS, 1982; GARCIA, 2004). Sobre o capital aplicado na produção incidiram os seguintes custos: juros e depreciação (amortização).

As estimativas de depreciação foram efetuadas pelo método linear (BUARQUE, 1991). A vida útil das instalações foi estimada em 30 anos, com valor residual de $30 \%$. A vida útil e o valor residual das máquinas foram estimados em 12 mil horas e $20 \%$ do valor inicial para os tratores, em 10 mil horas e $20 \%$ do valor inicial para as colheitadeiras e 8.000 horas e $10 \%$ do valor inicial para os equipamentos (distribuidor de calcário, pulverizador e semeadora), respectivamente (GADANHA; MOLIN; COELHO; YAHN; TOMIMORI, 1991). 
A remuneração do capital em instalações e equipamentos foi feita pela taxa de juros obtida pelos produtores nas linhas de financiamento para investimento em máquinas e equipamentos agrícolas. Segundo Hoffmann et al. (1987), costuma-se calcular os juros sobre o valor médio do capital empregado na atividade. $\mathrm{O}$ valor médio do bem é a média aritmética entre o valor inicial e o valor residual do bem de capital. O fluxo de serviço do capital é calculado somando-se os custos de juros e depreciação (amortização). O estoque de capital fixo é obtido multiplicando o valor inicial (novo) de máquinas, equipamentos e instalações por sua porcentagem de uso.

\subsubsection{Químicos (Q)}

Para composição dessa variável elaborou-se um índice considerando os gastos com herbicidas (dessecantes, pré e pós-emergentes), defensivos agrícolas (inseticidas, acaricidas, fungicidas e formicidas), adjuvantes, fertilizantes granulados e foliares. O índice foi composto por uma média ponderada, com fatores dados pelas parcelas de custo.

Os herbicidas são importantes na cultura da soja pelo controle de plantas daninhas que proporcionam. Essas invasoras competem com a soja por nutrientes durante o ciclo produtivo da cultura e diminuem o valor recebido por saco de soja colhido, por representarem impurezas no produto. Os defensivos são produtos químicos utilizados no combate e/ou prevenção de doenças e pragas agrícolas que incidem sobre as sementes e as plantas de soja causando queda na produção. As informações a respeito dos gastos com esses insumos químicos foram obtidas diretamente com os produtores pesquisados.

\subsubsection{Mão-de-obra (L)}

A variável mão-de-obra foi composta pelos gastos com a mão-deobra familiar e a contratada, incluindo os funcionários fixos nas propriedades e os temporários (diaristas). O preço da mão-de-obra ( $\mathrm{R}$ / hora) foi o quociente entre as despesas com este recurso (em R\$), incluindo os encargos sociais $(45,42 \%)$ sobre o salário, e o número total de horas trabalhadas. Os gastos totais com mão-de-obra foram obtidos diretamente da pesquisa de campo. 


\subsubsection{Terra (T)}

O valor do arrendamento foi considerado como uma proxy do preço da terra $(\mathrm{R} \$ / \mathrm{ha})$. O custo dessa parcela foi calculado considerando a área total plantada com soja na propriedade e o preço do arrendamento.

\subsubsection{Outros custos (O)}

Nesse item foram incluídos outros gastos que ocorrem na produção de soja: semente, tratamento de semente e operações com máquinas. Obteve-se o preço de aquisição da semente ( $\mathrm{R} \$ / \mathrm{kg}$ de semente) diretamente da pesquisa de campo. Para os produtores de soja transgênica que utilizaram semente própria, não certificada pelos órgãos nacionais competentes, considerou-se o preço comercial do grão de soja, referente ao mês de outubro de 2004. Para os produtores de soja não transgênica e transgênica que efetuaram a compra de sementes foram considerados os preços comerciais de aquisição das sementes. Não se considerou no preço da semente o custo da tecnologia transgênica.

Nos custos com tratamento de semente agregaram-se os gastos com inoculantes, micro nutrientes (cobalto e molibdênio), inseticidas e fun-

gicidas. Obteve-se o preço pago pelo tratamento de semente (em R $\$ / \mathrm{kg}$ de semente) pela divisão do gasto total com esses insumos (em R\$) pelo total de semente tratada utilizada (em kg).

Para o cálculo da variável operações com máquinas, as estimativas de conservação de máquinas e equipamentos foram efetuadas considerando-se uma taxa de manutenção de $50 \%$ sobre o valor inicial das máquinas (tratores e colheitadeiras), de $40 \%$ para distribuidores de calcário e pulverizadores e $80 \%$ para semeadoras. Calculou-se o consumo de combustível multiplicando-se a potência das máquinas pelo coeficiente 0,12 . O valor obtido multiplicado pelo preço do combustível forneceu o preço do consumo do combustível, em R $\$$ /hora.

\subsection{Estimativa do modelo}

Os fatores de produção considerados foram químicos $(\mathrm{Q})$, capital $(\mathrm{K})$, mão-de-obra (L), terra (T) e outros custos (O). O sistema de equações, sem restrições, composto pelas parcelas de custo é representado por: 


$$
\begin{aligned}
& S_{Q}=\beta_{Q}+\gamma_{Q Q} \ln P_{Q}+\gamma_{Q K} \ln P_{Q}+\gamma_{Q L} \ln P_{L}+\gamma_{Q T} \ln P_{T}+\gamma_{Q O} \ln P_{O}+{ }_{Q Y} \ln Y \\
& S_{K}=\beta_{K}+\gamma_{K Q} \ln P_{Q}+\gamma_{K K} \ln P_{K}+\gamma_{K L} \ln P_{L}+\gamma_{K T} \ln P_{T}+\gamma_{K O} \ln P_{O}+\gamma_{K Y} \ln Y \\
& S_{L}=\beta_{L}+\gamma_{L Q} \ln P_{Q}+\gamma_{L K} \ln P_{K}+\gamma_{L L} \ln P_{L}+\gamma_{L T} \ln P_{T}+\gamma_{L O} \ln P_{O}+\gamma_{L Y} \ln Y \\
& S_{T}=\beta_{T}+\gamma_{T Q} \ln P_{Q}+\gamma_{T K} \ln P_{K}+\gamma_{T L} \ln P_{L}+\gamma_{T T} \ln P_{L}+\gamma_{T O} \ln P_{O}+\gamma_{T Y} \ln Y \\
& S_{O}=\beta_{O}+\gamma_{O Q} \ln P_{Q}+\gamma_{O K} \ln P_{K}+\gamma_{O L} \ln P_{L}+\gamma_{O T} \ln P_{L}+\gamma_{O O} \ln P_{O}+\gamma_{O Y} \ln Y
\end{aligned}
$$

As parcelas de gastos com químicos, capital, mão-de-obra, terra e outros custos são representadas por $S_{Q}, S_{K}, S_{L}, S_{T}$ e $S_{O}$, respectivamente. Y é o nível da produção de soja (em toneladas) e $P_{Q}, P_{K}, P_{L}, P_{T}$ e $P_{O}$ são os preços dos insumos (em R\$).

As restrições de homogeneidade linear, dadas pelas equações (19) a (31) e as de simetria dadas pelas equações (32) a (41), são incorporadas ao sistema através da normalização das equações pelo preço da variável outros custos $\left(P_{O}\right)$ :

$$
\begin{aligned}
& \beta_{Q}+\beta_{K}+\beta_{L}+\beta_{T}+\beta_{O}=1 \\
& \gamma_{Q Y}+\gamma_{K Y}+\gamma_{L Y}+\gamma_{T Y}+\gamma_{O Y}=0 \\
& \gamma_{Q Q}+\gamma_{Q K}+\gamma_{Q L}+\gamma_{Q T}+\gamma_{Q O}=0 \\
& \gamma_{K Q}+\gamma_{K K}+\gamma_{K L}+\gamma_{K T}+\gamma_{K O}=0 \\
& \gamma_{L Q}+\gamma_{L K}+\gamma_{L L}+\gamma_{L T}+\gamma_{L O}=0 \\
& \gamma_{T Q}+\gamma_{T K}+\gamma_{T L}+\gamma_{T T}+\gamma_{T O}=0 \\
& \gamma_{O Q}+\gamma_{O K}+\gamma_{O L}+\gamma_{O T}+\gamma_{O O}=0 \\
& \gamma_{Q Q}+\gamma_{K Q}+\gamma_{L Q}+\gamma_{T Q}+\gamma_{O Q}=0 \\
& \gamma_{Q K}+\gamma_{K K}+\gamma_{L K}+\gamma_{T K}+\gamma_{O K}=0 \\
& \gamma_{Q L}+\gamma_{K L}+\gamma_{L L}+\gamma_{T L}+\gamma_{O L}=0
\end{aligned}
$$




$$
\begin{aligned}
& \gamma_{Q T}+\gamma_{K T}+\gamma_{L T}+\gamma_{T T}+\gamma_{O T}=0 \\
& \gamma_{Q O}+\gamma_{K O}+\gamma_{L O}+\gamma_{T O}+\gamma_{O O}=0 \\
& \gamma_{Q Q}+\gamma_{Q K}+\gamma_{Q L}+\gamma_{Q T}+\gamma_{Q O}+\gamma_{K Q}+\gamma_{K L}+\gamma_{K K}+\gamma_{K T}+\gamma_{K O}+\gamma_{L Q}+\gamma_{L K}+\gamma_{L L}+ \\
& \gamma_{L T}+\gamma_{L O}+\gamma_{T Q}+\gamma_{T K}+\gamma_{T L}+\gamma_{T T}+\gamma_{T O}+\gamma_{O Q}+\gamma_{O K}+\gamma_{O L}+\gamma_{O T}+\gamma_{O O}+ \\
& \gamma_{Q Q}+\gamma_{K Q}+\gamma_{L Q}+\gamma_{T Q}+\gamma_{O Q}+\gamma_{Q K}+\gamma_{K K}+\gamma_{L K}+\gamma_{T K}+\gamma_{O K}+ \\
& \gamma_{Q L}+\gamma_{K L}+\gamma_{L L}+\gamma_{T L}+\gamma_{O L}+\gamma_{Q T}+\gamma_{K T}+\gamma_{L T}+\gamma_{T T}+\gamma_{O T}+ \\
& \gamma_{Q K}=\gamma_{K Q} \\
& \gamma_{Q L}=\gamma_{L Q} \\
& \gamma_{Q T}=\gamma_{T Q} \\
& \gamma_{Q O}=\gamma_{O Q} \\
& \gamma_{K L}=\gamma_{L K} \\
& \gamma_{K T}=\gamma_{T K} \\
& \gamma_{K O}=\gamma_{O K} \\
& \gamma_{L T}=\gamma_{T L} \\
& \gamma_{L O}=\gamma_{O L} \\
& \gamma_{T O}=\gamma_{O T}
\end{aligned}
$$

Conforme já observado, como as parcelas dos gastos com os fatores químicos, capital, mão-de-obra, terra e outros custos somam-se à unidade $\left(S_{Q}+S_{K}+S_{L}+S_{T}+S_{O}=1\right)$, é necessário, também, suprimir a equação da parcela de gastos com outros custos, para evitar uma matriz de covariância singular. O modelo a ser estimado com as restrições impostas fica, assim, composto do seguinte conjunto de equações:

$$
\begin{aligned}
& S_{Q}=\beta_{Q}+\gamma_{Q Q} \ln \left(P_{Q} / P_{O}\right)+\gamma_{Q K} \ln \left(P_{K} / P_{O}\right)+\gamma_{Q L} \ln \left(P_{L} / P_{O}\right)+\gamma_{Q T} \ln \left(P_{T} / P_{O}\right)+\gamma_{Q Y} \ln Y \\
& S_{K}=\beta_{K}+\gamma_{K Q} \ln \left(P_{Q} / P_{O}\right)+\gamma_{K K} \ln \left(P_{K} / P_{O}\right)+\gamma_{K L} \ln \left(P_{L} / P_{O}\right)+\gamma_{K T} \ln \left(P_{T} / P_{O}\right)+\gamma_{K Y} \ln Y \\
& S_{L}=\beta_{L}+\gamma_{Q L} \ln \left(P_{Q} / P_{O}\right)+\gamma_{K L} \ln \left(P_{K} / P_{O}\right)+\gamma_{L L} \ln \left(P_{L} / P_{O}\right)+\gamma_{L T} \ln \left(P_{T} / P_{O}\right)+\gamma_{L Y} \ln Y \\
& S_{T}=\beta_{T}+\gamma_{Q T} \ln \left(P_{Q} / P_{O}\right)+\gamma_{K T} \ln \left(P_{K} / P_{O}\right)+\gamma_{L T} \ln \left(P_{L} / P_{O}\right)+\gamma_{T T} \ln \left(P_{T} / P_{O}\right)+\gamma_{T Y} \ln Y
\end{aligned}
$$


Os parâmetros excluídos do sistema são calculados por diferença de acordo com as expressões (46) a (52):

$$
\begin{aligned}
& \beta_{O}=1-\beta_{Q}-\beta_{K}-\beta_{L}-\beta_{T} \\
& \gamma_{O Y}=-\gamma_{Q Y}-\gamma_{K Y}-\gamma_{L Y}-\gamma_{T Y} \\
& \gamma_{Q O}=-\gamma_{Q Q}-\gamma_{Q K}-\gamma_{Q L}-\gamma_{Q T} \\
& \gamma_{K O}=-\gamma_{K Q}-\gamma_{K K}-\gamma_{K L}-\gamma_{K T} \\
& \gamma_{L O}=-\gamma_{L Q}-\gamma_{L K}-\gamma_{L L}-\gamma_{L T} \\
& \gamma_{T O}=-\gamma_{T Q}-\gamma_{T K}-\gamma_{T L}-\gamma_{T T} \\
& \gamma_{O O}=-\gamma_{O Q}-\gamma_{O K}-\gamma_{O L}-\gamma_{O T}
\end{aligned}
$$

\section{Resultados e Discussão}

Os resultados das estimativas das funções para as parcelas de custo, com a imposição das restrições de homogeneidade linear e simetria são apresentados na Tabela 1. As parcelas de custo calculadas pela média aritmética da amostra foram todas positivas, o que garante a monotonicidade da função (Figura 1). As maiores parcelas se referem às despesas com capital (36\%) e químicos (31\%). A participação é expressiva desses dois componentes no custo de produção da soja nos estados amostrados. O sistema de produção de soja é praticamente todo mecanizado, o que justifica a elevada participação do capital na composição do custo de produção dessa cultura.

As estimativas das elasticidades-preço direta e cruzada da demanda de fatores para a produção de soja, nos estados amostrados, estão na Tabela 2. Os sinais negativos das elasticidades-preço diretas, que compõem a diagonal principal, confirmam a concavidade da função custo. O somatório igual a zero das elasticidades-preço direta e cruzada, em cada linha indica a imposição da restrição de homogeneidade linear da função custo. A maior parte das elasticidades-preço diretas resultou em valores menores que a unidade, indicando que a demanda dos fatores analisados é inelástica. As elasticidades-preço diretas que apresentaram a menor sensibilidade 
na demanda de fatores são as referentes à mão-de-obra, ao capital e aos químicos. Para um aumento (diminuição) de $1 \%$ no preço da diária da mão-de-obra, a demanda por esse fator reduz (aumenta) 0,313\%. No caso dos químicos, para um aumento de $1 \%$ no seu preço, a demanda reduz-se em $0,538 \%$. Para terra, a elasticidade-preço direta apresentou maior valor em comparação aos fatores químicos, capital e mão-de-obra.

Tabela 1 - Resultados da estimativa das equações parciais de custo da soja, nos estados amostrados

\begin{tabular}{lccccccc}
\hline & \multicolumn{5}{c}{ Variáveis independentes (logaritmizadas) } \\
\cline { 2 - 7 } Parcelas & Intercepto & $\ln \mathrm{P}_{\mathrm{Q}}$ & $\ln \mathrm{P}_{\mathrm{K}}$ & $\ln \mathrm{P}_{\mathrm{L}}$ & $\ln \mathrm{P}_{\mathrm{T}}$ & $\ln \mathrm{P}_{\mathrm{O}}$ & $\ln \mathrm{Y}$ \\
\hline$S Q$ & $-0,451^{*}$ & $0,047^{*}$ & $-0,038^{* *}$ & 0,008 & $0,030^{* *}$ & $-0,048$ & $0,078^{*}$ \\
$S K$ & $1,343^{*}$ & $-0,037^{* *}$ & $0,051^{* *}$ & $-0,050^{*}$ & $-0,044^{* *}$ & 0,081 & $-0,111^{*}$ \\
$S L$ & $0,169^{*}$ & 0,008 & $-0,050^{*}$ & $0,031^{*}$ & $-0,011^{* *}$ & 0,022 & $-0,006^{*}$ \\
$S T$ & $0,001^{* *}$ & $0,030^{* *}$ & $-0,044^{* *}$ & $-0,011^{* *}$ & $0,002^{*}$ & 0,023 & $-0,000^{*}$ \\
$S O$ & $-0,063$ & $-0,048$ & 0,080 & 0,022 & 0,023 & $-0,077$ & 0,040 \\
Somatório & 1 & 0 & 0 & 0 & 0 & 0 & 0 \\
\hline
\end{tabular}

Nota: ${ }^{*}$ significativo a $1 \%,{ }^{* *}$ significativo a $5 \%,{ }^{* * *}$ significativo a $10 \%$.

$\mathrm{SQ}=$ parcela de custo referente aos químicos; $\mathrm{SK}$ = parcela de custo referente ao capital; $\mathrm{SL}$ = parcela de custo referente à mão-de-obra; $\mathrm{ST}=$ parcela referente à terra; $\mathrm{SO}=$ parcela referente a outros custos; $\ln Y=$ logaritmo da quantidade produzida; $\ln \mathrm{P}_{\mathrm{Q}}=$ logaritmo do preço dos químicos; $\ln \mathrm{P}_{\mathrm{K}}=$ logaritmo do preço do capital; $\ln \mathrm{P}_{\mathrm{L}}=$ logaritmo do preço da mão-de-obra; $\ln \mathrm{P}_{\mathrm{L}}=$ logaritmo do preço da terra e $\ln \mathrm{P}_{\mathrm{O}}=$ logaritmo do preço de outros custos, calculado pela restrição de homogeneidade.

Figura 1 - Parcelas médias de custo dos fatores de produção para a soja, nos estados amostrados

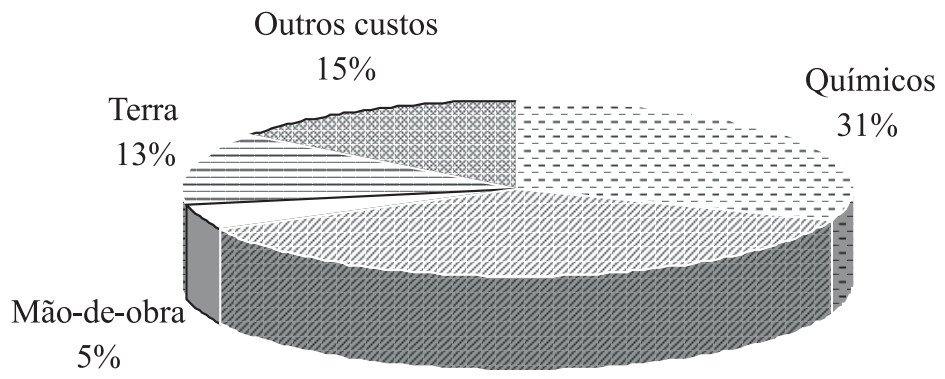
Capital
Fonte: dados da pesquisa 
Tabela 2 - Estimativas das elasticidades-preço diretas e cruzadas da demanda dos fatores para a produção de soja, nos estados amostrados.

\begin{tabular}{|c|c|c|c|c|c|c|}
\hline \multirow[b]{2}{*}{ Quantidade } & \multicolumn{5}{|c|}{ Preço dos fatores } & \multirow[b]{2}{*}{ Somatório } \\
\hline & Quimico & Capital & Mão-de-obra & Terra & Outros Custos & \\
\hline Quimico & $-0,538^{*}$ & $0,241^{*}$ & $0,076^{*}$ & 0,231 & $-0,011$ & 0 \\
\hline Capital & $\begin{array}{l}0,204 * \\
(4,466)\end{array}$ & $\begin{array}{l}-0,494^{*} \\
(-8,125)\end{array}$ & $\begin{array}{l}-0,090^{*} \\
(-2,858)\end{array}$ & $\begin{array}{c}0,012 \\
(0,023)\end{array}$ & 0,368 & 0 \\
\hline Mão-de-obra & $\begin{array}{l}0,483^{*} \\
(2,647)\end{array}$ & $\begin{array}{l}-0,670^{*} \\
(-2,858)\end{array}$ & $\begin{array}{l}-0,313^{*} \\
(-3,820)\end{array}$ & $\begin{array}{c}-0,091 \\
(-0,858)\end{array}$ & 0,592 & 0 \\
\hline Terra & $\begin{array}{c}0,536 \\
(0,505)\end{array}$ & $\begin{array}{c}0,032 \\
(0,023)\end{array}$ & $\begin{array}{c}-0,033 \\
(-0,858)\end{array}$ & $\begin{array}{l}-0,854^{*} \\
(-8,789)\end{array}$ & 0,319 & 0 \\
\hline Outros Custos & $-0,023$ & 0,918 & 0,198 & 0,291 & $-1,385$ & 0 \\
\hline
\end{tabular}

Nota: o valor de t encontra-se entre parênteses; *significativo a $1 \%{ }^{* *}$ significativo a $5 \%$ e ${ }^{* * *}$ significativo a $10 \%$.

Em relação às elasticidades-preço cruzadas, o sinal positivo indica substituição entre fatores e o sinal negativo complementaridade (Tabela 2). Os valores das elasticidades-preço cruzadas para a variável terra foram estatisticamente não significativos, o que tornou sua análise limitada. Observa-se que mão-de-obra e capital são fatores complementares: o aumento no preço de um fator leva à redução na demanda do outro fator. Terra e mão-de-obra e químicos e outros custos também apresentaram complementaridade entre si.

Nota-se relação de substituição entre todos os demais pares de fatores produtivos: químicos e capital, químicos e mão-de-obra, químicos e terra, capital e terra, capital e outros custos, mão-de-obra e outros custos e terra e outros custos. A relação de substituição indica que, à medida que o preço de um fator aumenta, o fator substituto é mais intensamente utilizado (ROCHELE; FERREIRA FILHO, 1999).

Parré e Ferreira Filho (1998) analisaram as relações de substituição entre os fatores de produção na cultura da soja no Estado de São Paulo e observaram que todos os fatores utilizados no processo produtivo apresentaram relações de substituição.

Os resultados das elasticidades de substituição parcial de Allen entre fatores estão na Tabela 3. Os valores fora da diagonal principal são simétricos e o valor positivo indica substituição entre fatores e o sinal negativo indica complementaridade. Na diagonal principal os valores referem-se às 
elasticidades de substituição diretas. Os sinais negativos das elasticidades de substituição diretas confirmam a concavidade da função custo.

Tabela 3 - Estimativas das elasticidades de substituição parcial de Allen entre fatores para produção de soja, nos estados amostrados

\begin{tabular}{|c|c|c|c|c|c|}
\hline \multirow[b]{2}{*}{ Quantidade } & \multicolumn{5}{|c|}{ Preço dos fatores } \\
\hline & Quimico & Capital & Mão-de-obra & Terra & $\overline{\text { Outros Custos }}$ \\
\hline Quimico & $-1,745$ & 0,662 & 1,566 & 1,739 & $-0,074$ \\
\hline Capital & & $-1,357$ & $-1,843$ & 0,088 & 2,524 \\
\hline Mão-de-obra & & & $-6,410$ & $-0,686$ & 4,062 \\
\hline Terra & & & & $-6,417$ & 2,189 \\
\hline Outros Custos & & & & & $-9,499$ \\
\hline
\end{tabular}

Fonte: dados da pesquisa

Observa-se que a maior parte das elasticidades na Tabela 3 apresentam sinais positivos, indicando substituição entre os fatores de produção de soja. Isto era de se esperar, pois estes sinais são determinados pelas elasticidades cruzadas. Os fatores químicos e mão-de-obra e químicos e terra apresentam relação de substituição e demanda relativamente elástica.

Os fatores capital e terra, bem como capital e outros custos também são considerados substitutos no processo produtivo. O valor da elasticidade de substituição parcial de Allen entre capital e terra é menor que a unidade. Quando a relação preço do capital/preço da terra aumenta $1 \%$, a quantidade relativa ótima (quantidade de terra/quantidade de capital) aumenta 0,08\% .

Mão-de-obra e capital são complementares na produção de soja. Para um aumento relativo de $1 \%$ no preço do capital, a razão quantidade de capital/mão-de-obra se reduz em 1,84\%. Pelo fato da Tabela 3 ser simétrica, a mesma relação é válida para mão-de-obra e capital. Há uma forte relação de complementaridade entre esses dois fatores, pois a variação relativa na demanda de um fator é mais que proporcional à variação no preço relativo entre eles. Os fatores mão-de-obra e terra e químicos e outros custos também são complementares.

Os resultados para as elasticidades de substituição de Morishima 
estão na Tabela 4. Os valores representam as elasticidades de substituição do fator que se encontra na linha em relação ao fator da coluna, com variação do preço do fator da coluna. O conceito de Morishima não é simétrico e é menos restritivo, pois relaciona a variação nas quantidades relativas desses fatores com o ajuste ótimo de ambos para variações no preço de um deles.

De acordo com a classificação de Allen, os fatores mão-de-obra e capital são complementares. Na classificação de Morishima, eles serão complementares quando o preço de capital variar $\left(\sigma_{i j}^{M}=-0,1766\right)$ e substitutos quando variar o preço do fator mão-de-obra $\left(\sigma_{i j}^{M}=0,2234\right)$. À medida que o preço da mão-de-obra aumenta, diminui a utilização de capital (comportamento complementar), porém isso também provoca o declínio na utilização da mão-de-obra devido à concavidade da função custo nos preços dos fatores, mas a uma taxa que faz com que a relação entre a utilização dos fatores capital/mão-de-obra cresça, o que evidencia substituição. Esse efeito não é captado pelo conceito de Allen.

Tabela 4 - Estimativa das elasticidades de substituição de Morishima, entre os fatores de produção para a cultura da soja, nos estados amostrados.

\begin{tabular}{lccccc}
\hline & Quimico & Capital & Mão-de-obra & Terra & Outros Custos \\
\hline Quimico & & 0,735 & 0,390 & 1,086 & 1,375 \\
Capital & 0,742 & & 0,223 & 0,866 & 1,754 \\
Mão-de-obra & 1,021 & $-0,177$ & & 0,763 & 1,978 \\
Terra & 1,074 & 0,526 & 0,280 & & 1,705 \\
Outros Custos & 0,515 & 1,412 & 0,512 & 1,146 & \\
\hline
\end{tabular}

Fonte: dados da pesquisa

\section{Conclusões}

Este estudo estimou as parcelas de custo para a produção de soja para os estados de Mato Grosso, Mato Grosso do Sul, Goiás, Paraná e Rio Grande do Sul, através do modelo translog. As despesas com os fatores capital e químicos representaram as maiores parcelas dos custos de produção.

As estimativas das elasticidades-preço diretas indicaram que os fatores considerados possuem demanda inelástica, com exceção de outros 
custos. A demanda por terra foi a mais sensível às variações no preço. As elasticidades-preço cruzadas mostraram que há complementaridade entre os fatores mão-de-obra e capital. A relação de substituição predominou entre os demais fatores produtivos. Quanto ao fator terra, como os seus valores de elasticidades-preço cruzadas apresentaram-se não significativos estatisticamente, sua análise ficou limitada.

As elasticidades de substituição parcial de Allen indicaram substituição entre a maior parte dos fatores de produção de soja. Houve uma forte relação de complementaridade entre os fatores capital e mão-deobra e de substituição entre os fatores químicos e mão-de-obra. Na classificação de Morishima, capital e mão-de-obra são complementares quando o preço de capital varia e substitutos quando varia o preço do fator mão-de-obra.

\section{Referências bibliográficas}

ALVES, E. A função custo. Piracicaba: ESALQ, DESR, 1995. 99 p. (Série didática, 102).

BAIRAM, E.I. (Ed.). Production and cost functions: specification, measurement and applications. Vermont: Ashgate Publ., 1998. 132 p.

BAIRAM, E.I.; KALYA, E. Production versus cost functions: unreliability of the duality theorem in accouting and economics. In: BAIRAM, E.I. (Ed.). Production and cost functions: specification, measurement and applications. Vermont: Ashgate Publ., 1998. p. 42-53.

BEATTIE, B.R.; TAYLOR, C.R. The economics of production. New York: John Wiley, 1985. $258 \mathrm{p}$.

BERNDT, E.R.; WOOD, D.O. Technology, prices and the derived demand for energy. The Review of Economics and Statistics, Cambridge, v. 57, n. 3, p. 259-268, Aug. 1975.

BINSWANGER, H.P. A cost function approach to the measurement of elasticities of factor demand and elasticities of substitution. American Journal of Agricultural Economics, Saint Paul, v. 56, p. 377 - 386, May 1974. 
BLACKORBY, C; RUSSEL, R.R. The Morishima elasticity of substitution, symmetry, constancy, separability and its relationship to the Hicks and Allen elasticities. Review of Economic Studies, Bristol, v.48, p. 147-158, 1981.

BRASIL. Ministério do Desenvolvimento, Indústria e Comércio Exterior. Alice Web. Disponível em: http://aliceweb.desenvolvimento.gov. br/alice.asp. Acesso em: 12 jul. 2005.

BUARQUE, C. Avaliação econômica de projetos. Rio de Janeiro: Campus, 1984. $266 \mathrm{p}$.

CHAMBERS, R. G. Applied production analysis: a dual approach. New York: Cambridge University Press, 1998. 331 p.

CHRISTENSEN, L.R.; GREENE, W.H. Economies of Scale in U.S. Electric Power Generation. Journal of Political Economy, Chicago, v. 84, n. 4, p. 655-676, 1976.

COMPANHIA NACIONAL DE ABASTECIMENTO. Disponível em: http:// www.conab.com.br/. Acesso em: 15 set. 2005.

CONFEDERAÇÃO NACIONAL DA AGRICULTURA E PECUÁRIA DO BRASIL. Disponível em: http://www.cna.org.br/. Acesso em: 15 set. 2005.

DIAS, R.S. Elasticidade de substituição e de demanda de fatores na agricultura brasileira. 1982. 58 p. Dissertação (Mestrado em Economia Rural) - Universidade Federal de Viçosa, Viçosa, 1982.

FAO. Statistical databases. Disponível em: http://www.fao.org/. Acesso em: 20 ago. 2005.

FIGUEIREDO, M. G. de. Agricultura e estrutura produtiva do Estado do Mato Grosso: uma análise insumo-produto. 187p. Dissertação (Mestrado em Economia Aplicada) - Escola Superior de Agricultura "Luiz de Queiroz", Universidade de São Paulo. Piracicaba, 2003.

GADANHA, D.C.; MOLIN, J.P.; COELHO, J.L.D.; YAHN, C.H.; TOMIMORI, S.M.A.W. Máquinas e implementos agrícolas do Brasil. São Paulo: IPT, 1991. 468 p.

GARCIA, L.A.F. Economias de escala na produção de frangos de cortes no Brasil. 2004. 114 p. Tese (Doutorado em Economia Aplicada) 
- Escola Superior de Agricultura "Luiz de Queiroz”, Universidade de São Paulo, Piracicaba, 2004.

GOMES, M.F.M.; ROSADO, P.L. Mudança na produtividade dos fatores de produção da cafeicultura nas principais regiões produtoras do Brasil. Revista de Economia e Sociologia Rural, Brasília, v. 43, n. 4, p. 633655, out/dez. 2005.

GREENE, W.H. Econometric analysis. 4th ed. Inc. Upper Saddle River: Prentice-Hall, 2000. 1004 p.

HOFFMANN, R.; ENGLER, J.J. de C.; SERRANO, O. Administração da empresa agrícola. 2.ed. São Paulo: Pioneira, 1987. 325 p.

LERDA, J.C. Resultados básicos na teoria da dualidade: vantagens e alguns usos em microeconomia. Estudos Econômicos, São Paulo, v. 9, n. 1, p.101 - 133, jan./abr. 1979.

LERNER, A.P. The question of symmetry. Review of Economic Studies, Bristol, v. 1, p.147-148, 1934.

LIMA, J.E. de. Definições de elasticidades de substituição: revisão e aplicação. Revista Brasileira de Economia e Sociologia Rural, Brasília, v. 38, n. 1, p. 9-44, jan./mar. 2000.

MILAN, M. Gestão sistêmica e planejamento de máquinas agrícolas. 2004. 100 p. Tese (Livre-Docência) - Escola Superior de Agricultura “Luiz de Queiroz”, Universidade de São Paulo, Piracicaba, 2004.

PARRÉ, J.L.; FERREIRA FILHO, J.B.S. Estudo da tecnologia utilizada na produção de soja no Estado de São Paulo. Revista Teoria e Evidência Econômica, Passo Fundo, v.6, n.11, p.7-18, nov. 1998.

RAY, S.C. A translog cost function analysis of U.S agriculture, 1939-77. American Journal of Agricultural Economics, Saint Paul, v. 56, p. 490-498, 1982.

REIS, R.P.; TEIXEIRA, E.C. Estrutura de demanda e substituição de fatores produtivos na pecuária leiteira: o modelo de custo translog. Revista Brasileira de Economia, Rio de Janeiro, v. 49, n. 3, p.545-554, jul/set. 1995. ROCHELLE, T.C.P.; FERREIRA FILHO, J.B.S. Função de custo translog e 
o mercado de fatores para o algodão no Estado de São Paulo: o caso da DIRA de Campinas. Revista de Economia e Sociologia Rural. Brasília, v. 37, n. 2, p.77-95, abr./jun. 1999.

UZAWA, H. Production functions with constant elasticities of substitution. Review of Economics Studies, Clevedon, v.29, n. 81, p. 291-299. 1962.

ZAGATTO, L.C.A.G. Estrutura produtiva de pequenos agricultores e implicações para a geração e adoção de tecnologia. 1991. 122p. Tese (Doutorado em Economia Rural) - Universidade Federal de Viçosa, Viçosa, 1991.

ZELLNER, A. Na efficient method of estimating seemingly unrelated regressions and tests for aggregattion bias. Journal of the American Statistical Association. New York, n. 57, p. 349-368, June 1962.

Recebido em julho de 2006 e revisto em dezembro de 2006 